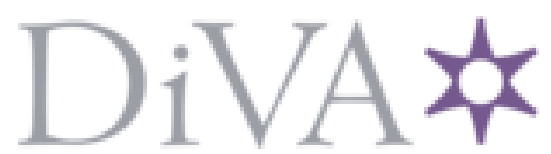

http://www.diva-portal.org

\title{
Postprint
}

This is the accepted version of a paper presented at IEEE PowerTech Conference.

Citation for the original published paper:

Singh, R S., Hooshyar, H., Vanfretti, L. (2017)

Testing and analysis of centralized and decentralized mode estimation architectures for active distribution network monitoring.

In:

N.B. When citing this work, cite the original published paper.

Permanent link to this version:

http://urn.kb.se/resolve?urn=urn:nbn:se:kth:diva-205285 


\section{Testing and Analysis of Centralized and Decentralized Mode Estimation Architectures for Active Distribution Network Monitoring}

\author{
Ravi Shankar Singh \\ Electrical Energy Systems \\ TU Eindhoven \\ Eindhoven, NL \\ Email: r.singh1@tue.nl
}

\author{
Hossein Hooshyar \\ Electric Power and Energy Systems \\ KTH Royal Institute of Technology \\ Stockholm, Sweden \\ Email: hosseinh@kth.se
}

\author{
Luigi Vanfretti \\ KTH Royal Institute of Technology \\ Stockholm, Sweden \\ Email: luigiv@kth.se
}

\begin{abstract}
This paper presents the results of testing a Phasor Measurement Unit (PMU) data-based mode estimation application deployed within a decentralized architecture using a realtime test platform. By extending the author's previous work in [1], this paper shows that a decentralized architecture is effective in detecting local modes which are less observable in presence of other dominant modes when estimated in a centralized architecture. The tests in this paper were carried out that was coupled with actual PMUs, a Phasor Data Concentrator (PDC) and two types of mode estimation architectures. The is network comprised of a high voltage network connected to an Active Distribution Network (ADN) with Renewable Energy Resources (RES). In the decentralized architecture where each PMU was associated with its own processing unit running to compute mode estimates from the acquired time-series data. The results of the decentralized mode estimation architecture are analyzed and compared with centralized architecture.
\end{abstract}

Index Terms-Power System Monitoring, Phasor Measurement Unit (PMU), Mode-meter, Decentralized Mode Estimation, Oscillations.

\section{INTRODUCTION}

Wide-Area Monitoring System (WAMS) applications have been developed to acquire critical information about a network's dynamics. Modal frequencies and damping ratios are useful indicators of power system stress, which usually deteriorates with increased burden on the system. Real-time estimation of these indicators from continuous time-series measurements have become the base for synchrophasor-based real-time power system monitoring and early warning systems, including applications implemented in WAMS for real-time monitoring purposes [2], [3] and [4].

In practice, today's applications utilize a centralized architecture where a central processor acquires data from all the connected PMUs. Acquired data is processed and fed to applications (such as mode estimators that provide estimates

\footnotetext{
This work was supported in part by the FP7 IDE4L project funded by the European Commission, the STandUp for Energy Collaboration Initiative and by Statnett SF, the Norwegian TSO. Website: http://ide4l.eu/.

Ravi Shankar Singh is supported by the European Union's Horizon 2020 research and innovation programme under the Marie Sklodowska-Curie grant agreement No 676042 .
}

of modal parameters). Although [3] indicates that other architectures can aid in the performance of the mode-meter applications, no experimental testing (real-time, PMU-in-theloop) or field implementations have been implemented. In [1], the authors have shown the benefits of using a decentralized architecture by analyzing synthetic PMU data. This paper aims to expand the study of the decentralized architecture and mode estimation application by performing experimental laboratory tests including commercial PMUs interfaced with a real-time simulator running an active distribution network model.

The remainder of the paper is arranged as follows: A brief introduction of the mode estimator is given in Section II. Section III describes the test system. Section IV identifies the modes present in the system using spectral analysis and Section V presents the results. Conclusions are drawn in Section VI.

\section{Mode Estimator Application}

The application employs measurement-based system identification methods to estimate a power system's modal parameters. In quasi steady state, the application acquires 'ambient' data, and runs an Auto-Regressive Moving Average (ARMA)based Modified Yule Walker method to estimate modal parameters. The application acquires PMU data using the $S^{3} \mathrm{DK}$ toolkit within the LabVIEW platform [5] and its source code can be found on Github [6]. The $S^{3} \mathrm{DK}$ acts as a data mediator receiving IEEE C37.118.2 formatted signals and converting them into LabVIEW data-types.

This paper tests a decentralized architecture for mode estimation where system modes are estimated by local, individual processors using single PMU data streams. This is different than the centralized estimator where all the different PMU data streams are processed by a single central processing unit. The locally estimated modes can be collected and sent to higher level aggregators. This architecture aims to increase identification capability of localized oscillations that might not be observed by centralized architecture. For testing and comparison purposes, the mode estimator application was run using both architectures. 


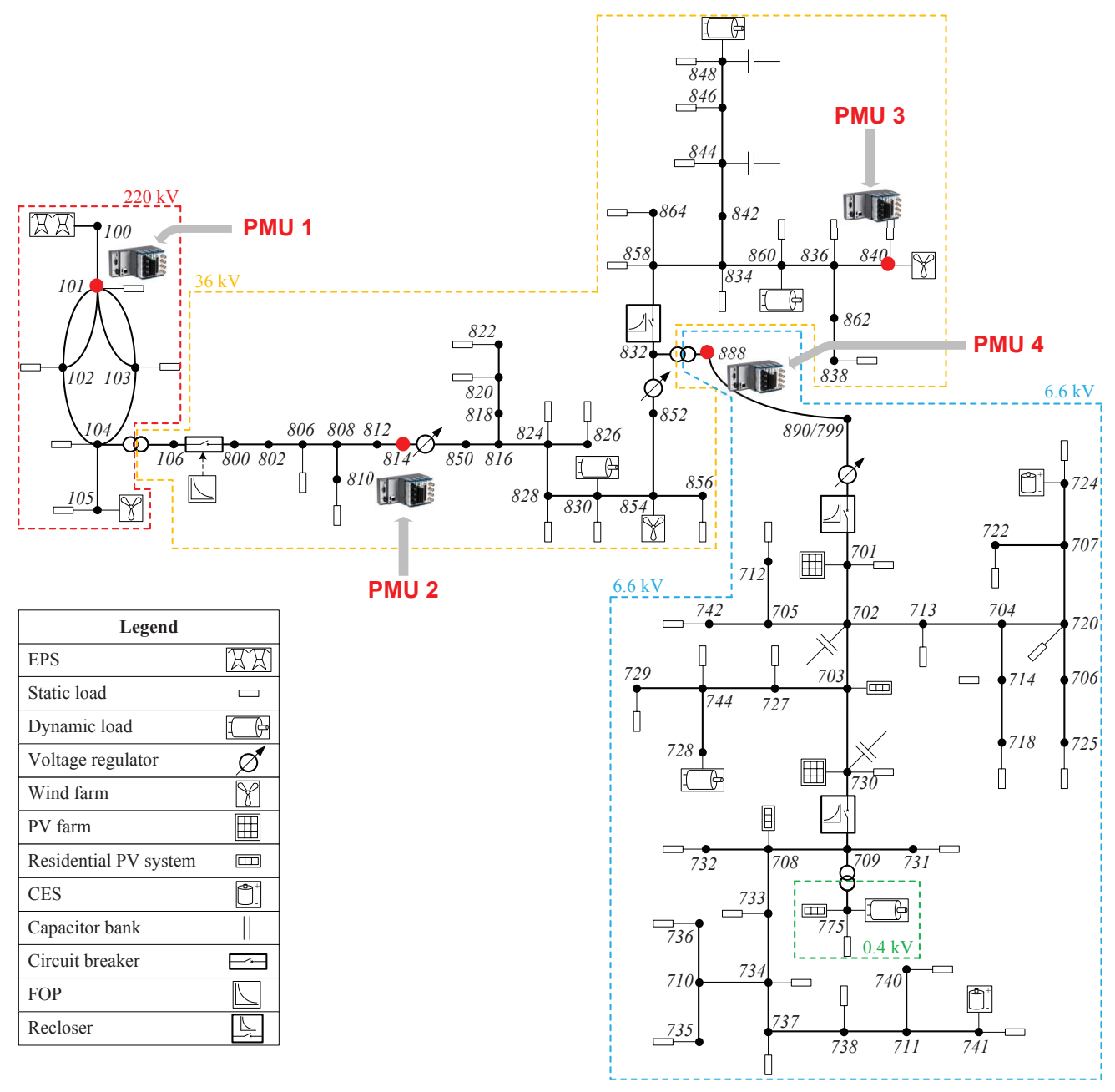

Fig. 1. Test grid model with marked PMU locations.

\section{Test System}

The test grid includes a high-voltage (HV) transmission grid along with a highly active distribution grid at mediumvoltage (MV) that includes distributed generation in form of wind farms and solar parks. More information about the grid can be found in [7] and [8]. As shown in Figure 1, four different measurement locations at the HV, MV and lowvoltage (LV) levels were equipped with PMUs to acquire synchrophasor data. The model was simulated using a realtime simulator. Measured PMU data from nodes $101(\mathrm{HV})$, $814(\mathrm{MV}), 840(\mathrm{MV})$ and $888(\mathrm{LV})$ were streamed to a PDC.

\section{SPECTRAL ANALYSiS}

To assess the results from the mode-meter application using PMU data, baseline values of the modal frequencies were obtained through spectral analysis. First the Fast Fourier Transform (FFT) was used to identify the inter-area and/or local modes present in the different measured signals including voltage magnitude, current magnitude and voltage angle difference signals generated through offline simulation.

To induce inter-area oscillations, the inertia of the synchronous generator at bus 100 was decreased gradually, thus weakening the system and making the main inter-area mode visible. The FFT analysis identified the frequency of the oscillations to be $0.41 \mathrm{~Hz}$. This inter-area oscillation was present throughout the grid. To illustrate the advantages of decentralized mode-estimation in identifying local oscillations, a localized forced oscillation was injected by introducing a
TABLE I

INTER-AREA AND FORCED LOCAL MODES IN THE GIRD

\begin{tabular}{|c|c|}
\hline Mode & Frequency \\
\hline Mode 1 (inter-area) & $0.41 \mathrm{~Hz}$ \\
\hline Mode 2 (forced local) & $1.7 \mathrm{~Hz}$ \\
\hline
\end{tabular}




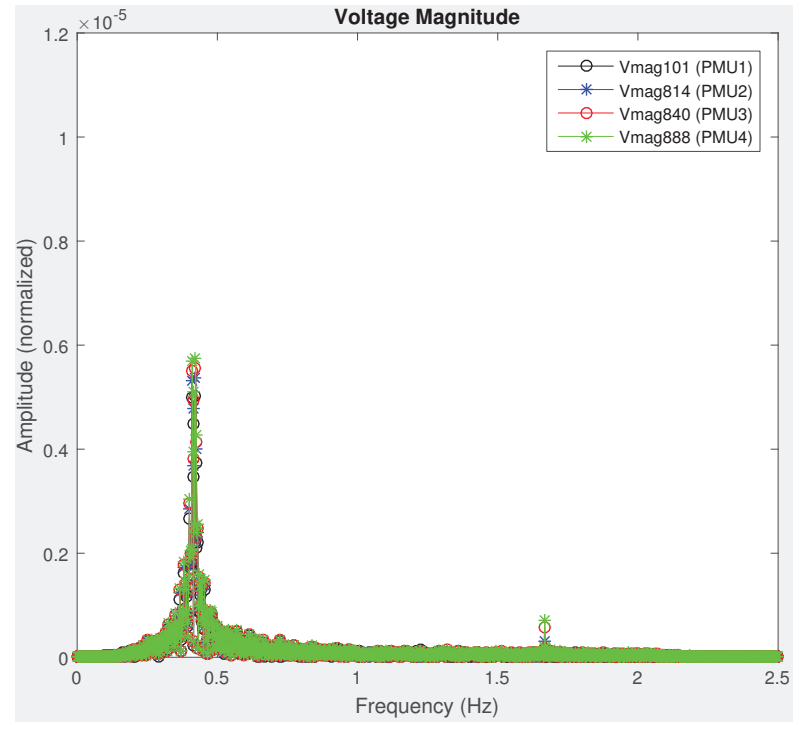

Fig. 2. Frequency spectra plot using the voltage magnitude estimates from the PMUs at nodes $101,814,840$ and 888 as shown in Fig. 1.

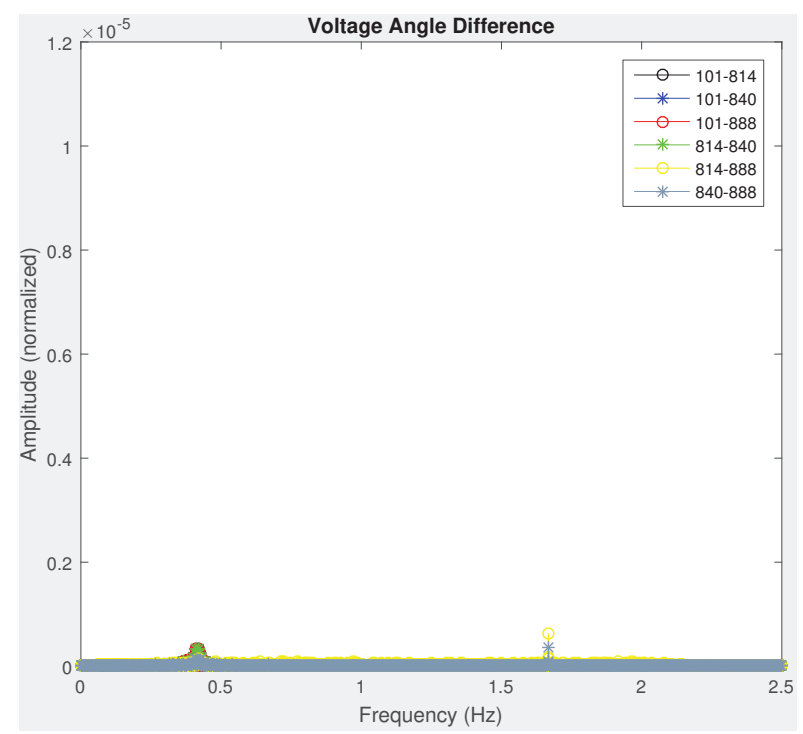

Fig. 4. Frequency spectra plot using the voltage angle difference estimates from the PMUs at nodes 101, 814, 840 and 888 as shown in Fig. 1.

sinusoidal load variation at node 888 in the LV section with a frequency of $1.7 \mathrm{~Hz}$. Table I presents the frequency of both the modes to be identified Through spectral Analysis.

Next, the model was run in real-time and data was acquired using four PMUs. The FFT analysis of the PMU data also confirmed the presence of an inter-area mode of frequency $0.41 \mathrm{~Hz}$ as identified earlier in the previous step. Figures 2 and 3 show the estimated frequency spectrum in the voltage and current magnitude signals from PMUs at different nodes. Figure 4 presents estimated frequency spectra using the differences between the measured angles at respective nodes. The frequency spectra are normalized by dividing all the

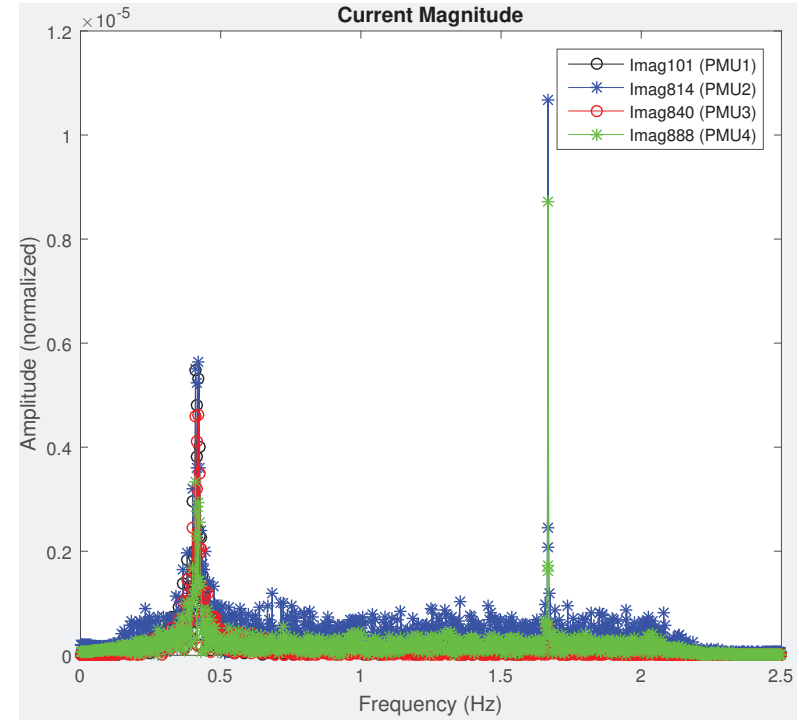

Fig. 3. Frequency spectra plot using the current magnitude estimates from the PMUs at nodes 101, 814, 840 and 888 as shown in Fig. 1.

measurement vectors with respect to their Eucledian Norms. Presence of two modes is observed in all the three spectrum plots.

The results shown above are necessary to configure the mode meter application, which requires a frequency band for each mode to be estimated. With these results, the application was utilized to estimate the modal frequencies and associated damping ratios. The results are presented in the next section. For comparison purposes, the real-time experiments are run for both the centralized and decentralized architectures.

\section{RESULTS}

This section presents the results obtained by the modemeter application running in real-time. Results obtained from both architectures are presented for comparison and analysis. Estimates were calculated on a moving window of ten minutes of data. The sampling rate was $10 \mathrm{~Hz}$, so the window contained 6000 samples. Each test was run to obtain 1400 estimates. The estimated results were stored for further analysis. Probability Distribution Function (PDF) plots were plotted for estimated frequencies and damping ratios. PMU estimates of voltage magnitudes and voltage angle difference are used as input signals to the application. Both cases are discussed below.

TABLE II

STATISTICAL ANALYSIS OF CENTRALIZED MODE ESTIMATION BY PROCESSING ALL THE VOLTAGE MAGNITUDE SIGNALS TOGETHER

\begin{tabular}{cccccccc}
\hline \multicolumn{3}{c}{ Mode 1 } & \multicolumn{4}{c}{ Mode 2 } \\
\hline \multicolumn{2}{c}{ Freq. (Hz) } & \multicolumn{2}{c}{ Damp. (\%) } & \multicolumn{2}{c}{ Freq. (Hz) } & Damp. (\%) \\
\hline$\mu^{a}$ & $\sigma^{b}$ & $\mu$ & $\sigma$ & $\mu$ & $\sigma$ & $\mu$ & $\sigma$ \\
\hline .408 & .005 & 3.05 & 2.28 & 1.702 & .096 & 2.89 & 3.05 \\
\hline
\end{tabular}

${ }^{a} \mu$ : mean

${ }^{b} \sigma:$ Standard deviation 


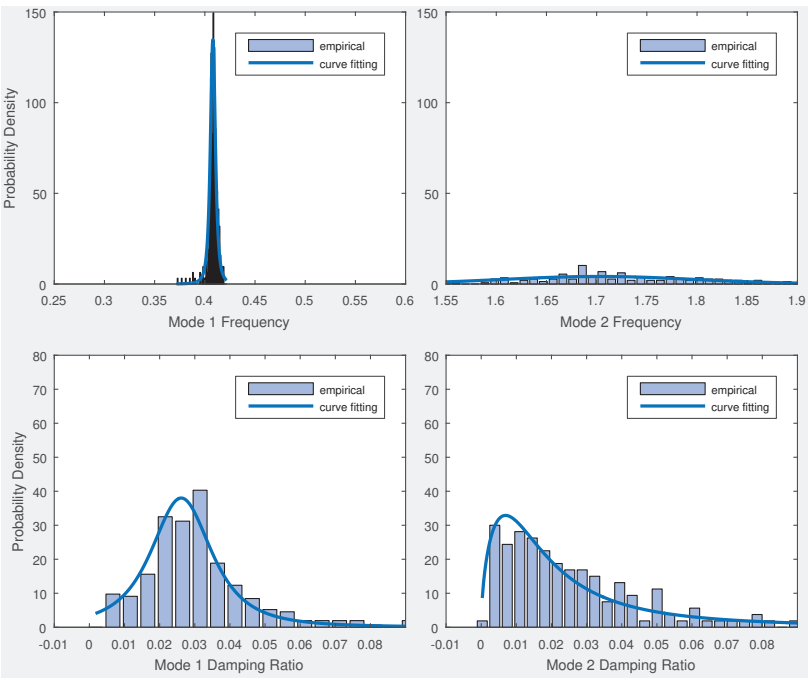

Fig. 5. Estimates from centralized architecture using voltage magnitude signals; TOP: PDF of Mode 1 frequency (left), PDF of Mode 2 frequency (right); BOTTOM: PDF of Mode 1 damping ratio (left), PDF of Mode 2 damping ratio (right).

\section{A. Voltage Magnitude Signals}

Positive sequence voltage phasor magnitudes acquired from the four PMUs were used as input signals for the mode-meter application. PDFs of the estimated frequencies and damping ratios were obtained. Results from centralized estimation architecture show high density of estimates around mode 1 with mean frequency and standard deviation [0.408, 0.005] Hz. For mode 2, the mean frequency and standard deviation of estimates are [1.702, 0.096] Hz. The mean and standard deviation of the frequency and damping of the two modes identified are presented in Table II. The summary of results in terms of PDF plots for centralized architecture is presented in Fig. 5.

From the PDF plots, it can be observed that, in the case of voltage magnitude signals and centralized architecture, the estimates have a very high probability density function for Mode 1 which peaks to about 150 at $0.41 \mathrm{~Hz}$. The distribution is Gaussian with standard deviation of $0.005 \mathrm{~Hz}$ around the mean frequency. The peak of probability density function for Mode 2 is 12 near the mean $1.702 \mathrm{~Hz}$. Hence the PDF peak is lower for mode 2 and the distribution has a comparatively

TABLE III

STATISTICAL ANALYSIS OF DECENTRALIZED MODE ESTIMATION BY PROCESSING ALL THE VOLTAGE MAGNITUDE SIGNALS INDIVIDUALLY

\begin{tabular}{ccccccccc}
\hline & \multicolumn{4}{c}{ Mode 1 } & \multicolumn{3}{c}{ Mode 2 } \\
\cline { 2 - 9 } Singnal & \multicolumn{1}{c}{ Freq. (Hz) } & \multicolumn{1}{c}{ Damp. (\%) } & \multicolumn{1}{c}{ Freq. (Hz) } & \multicolumn{2}{c}{ Damp. (\%) } \\
\cline { 2 - 9 } & $\mu$ & $\sigma$ & $\mu$ & $\sigma$ & $\mu$ & $\sigma$ & $\mu$ & $\sigma$ \\
\hline PMU 1 & .4081 & .0040 & 3.11 & 1.14 & 1.819 & .135 & 6.83 & 4.91 \\
PMU 2 & .4084 & .0039 & 3.15 & 1.02 & 1.720 & .087 & 5.03 & 5.35 \\
PMU 3 & .4081 & .0038 & 3.14 & 0.93 & 1.719 & .050 & 2.20 & 4.19 \\
PMU 4 & .4079 & .0038 & 3.13 & 0.80 & 1.721 & .048 & 2.41 & 5.88 \\
\hline
\end{tabular}

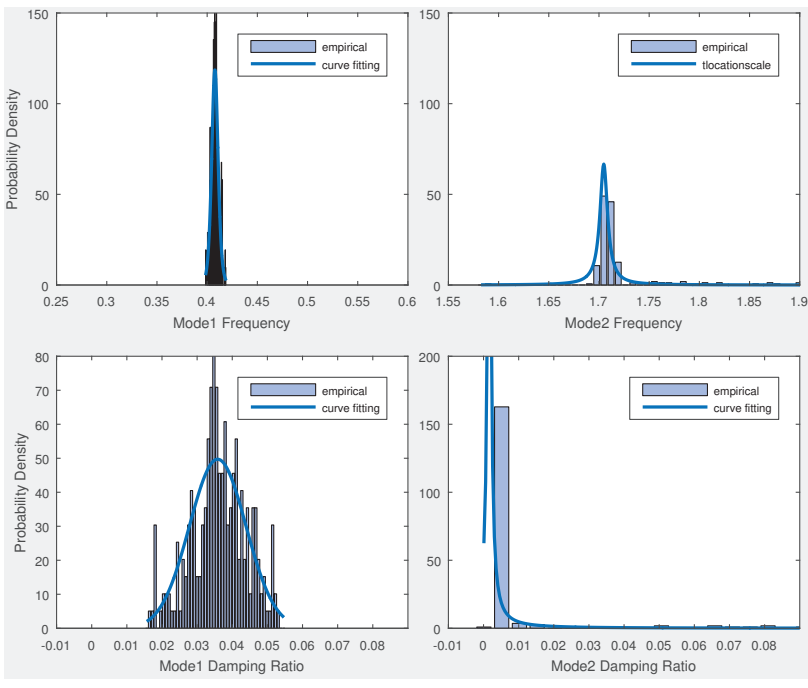

Fig. 6. Estimates from decentralized architecture using voltage magnitude signals from PMU4 placed at node 888 in the network; TOP: PDF of Mode 1 frequency (left), PDF of Mode 2 frequency (right); BOTTOM: PDF of Mode 1 damping ratio (left), PDF of Mode 2 damping ratio (right).

higher standard deviation of $0.096 \mathrm{~Hz}$.

Next the decentralized mode estimator results are analyzed. All the PMU data streams were processed individually. Using the data from PMU 4 at node 888, mean frequency and standard deviation calculated for mode 1 are [0.4079, 0.0038] $\mathrm{Hz}$ and for mode 2 are [1.721, 0.048] Hz. Similarly, mean damping ratio and standard deviation calculated for mode 1 are $[3.13,0.80] \%$ and for mode 2 are $[2.41,5.88] \%$.

Figure 6 presents the PDF plots of frequency and damping ratio estimates in the case of decentralized architecture using voltage magnitude signals. The plots show high probability density of estimates around mode 1 with mean frequency of $0.4079 \mathrm{~Hz}$. The probability distribution for mode 1 is quite similar to what was observed in the centralized cases. Still, the variance in frequency and damping ratio estimates in decentralized architecture are smaller than the variance observed by centralized architecture.

However, the PDF for mode 2 in the decentralized case has improved drastically. The peak of the PDF has increased from about 12 to 50 and the standard deviation of estimates around the mean frequency has decreased from $0.096 \mathrm{~Hz}$ to 0.048 Hz. This means more number of accurate estimates for mode 2 have been observed by the application in decentralized architecture running at local level. One interesting point to realize here is that, for mode 2 , the variance in frequency estimates is smaller in decentralized architecture but the variance in damping is higher when compared to centralized architecture. This indicates forced oscillation. Mean values and standard deviations of frequency and damping ratio estimates of the two modes identified by decentralized architecture using voltage magnitude from all the PMUs are presented in Table III. 


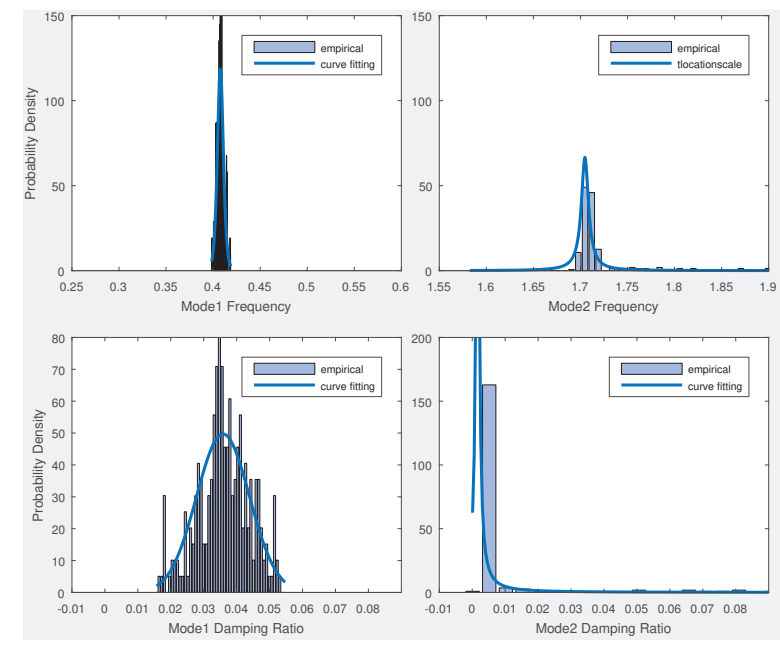

Fig. 7. Estimates from centralized architecture using all four voltage angle difference; TOP: PDF of Mode 1 frequency (left), PDF of Mode 2 frequency (right); BOTTOM: PDF of Mode 1 damping ratio (left), PDF of Mode 2 damping ratio (right).

\section{B. Voltage Angle Difference}

In this case, voltage angles difference between the measured angles at different nodes were used as input signals to the mode-meter application. Four combinations of voltage angle difference signals were formed: PMU1-PMU2, PMU1-PMU3, PMU1-PMU4 and PMU3-PMU4.

In the case of the centralized architecture, these signals were processed by a central processor. The estimates again have a very high probability density function for Mode 1 which peaks near the mean frequency of $0.414 \mathrm{~Hz}$ to about 150 . The distribution is Gaussian with standard deviation of 0.004 $\mathrm{Hz}$ around the mean frequency. The standard deviation of frequency estimates around the mean of mode 1 is $0.004 \mathrm{~Hz}$. The peak of probability density function for Mode 2 is near the mean $1.699 \mathrm{~Hz}$ to about 50, which is comparatively lower than the mode 1's frequency PDF but higher than what was observed for mode 2 in case of voltage magnitude signals in the centralized architecture. The Gaussian distribution has a comparatively higher standard deviation of $0.052 \mathrm{~Hz}$.

The mean and standard deviation of the frequency and damping of the two modes identified by centralized architecture are presented in Table IV. The PDF plot based on centralized estimation is presented in Fig. 7.

The last test was to implement the decentralized architecture using voltage angle difference signals. Each difference signal was processed individually by local processors. Results using

TABLE IV

STATISTICAL ANALYSIS OF CENTRALIZED MODE ESTIMATION PROCESSING ALL THE VOLTAGE ANGLE DIFFERENCE SIGNALS TOGETHER

\begin{tabular}{cccccccc}
\hline \multicolumn{3}{c}{ Mode 1 } & \multicolumn{4}{c}{ Mode 2 } \\
\hline \multicolumn{2}{c}{ Freq. (Hz) } & \multicolumn{2}{c}{ Damp. (\%) } & \multicolumn{2}{c}{ Freq. (Hz) } & \multicolumn{2}{c}{ Damp. (\%) } \\
\hline$\mu$ & $\sigma$ & $\mu$ & $\sigma$ & $\mu$ & $\sigma$ & $\mu$ & $\sigma$ \\
\hline .414 & .004 & 2.85 & 2.28 & 1.699 & .052 & 2.75 & 5.05 \\
\hline
\end{tabular}

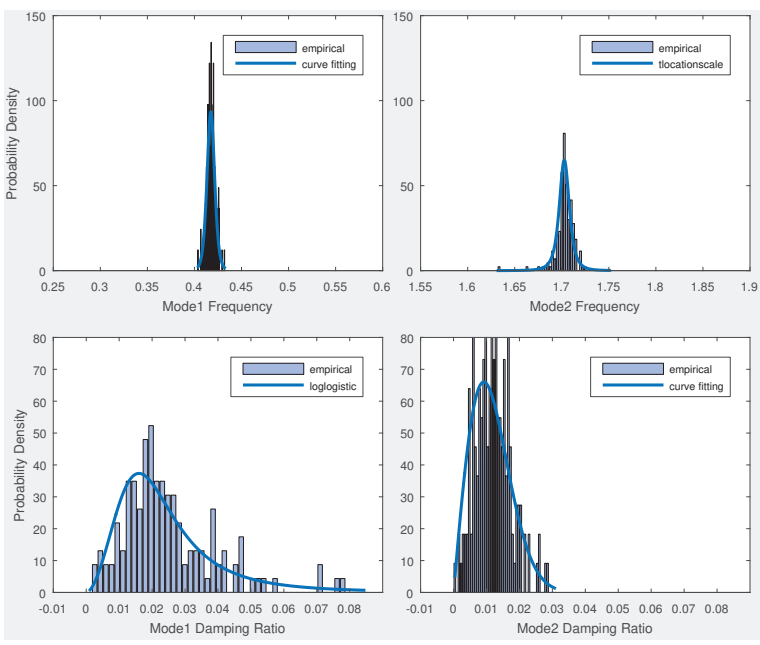

Fig. 8. Estimates from decentralized architecture using voltage angle difference between PMU3 and PMU4; TOP: PDF of Mode 1 frequency (left), PDF of Mode 2 frequency (right); BOTTOM: PDF of Mode 1 damping ratio (left), PDF of Mode 2 damping ratio (right).

voltage angle difference between PMU3 and PMU4, achieved mean frequency and standard deviation of [0.4175, 0.0049] Hz for mode 1 and [1.7021, 0.048] Hz for mode 2.

Fig. 8 presents the PDF plots of frequency and damping ratio estimates in case of decentralized architecture using voltage angle difference signals. The plots show PDF peak of about 130 around mode 1 with mean frequency of 0.4175 $\mathrm{Hz}$. The PDF for mode 2 in the decentralized case has improved by some margin. The peak of the PDF has increased from about 45 to 75 and the standard deviation of estimates around the mean frequency has decreased from $0.052 \mathrm{~Hz}$ to $0.048 \mathrm{~Hz}$. Mean values and standard deviations of frequency and damping ratio estimates of the two modes identified by decentralized architecture using voltage angle difference between PMU 3 and PMU 4 are presented in Table V.

The results show that, for centralized architecture, voltage angle difference signals are more suitable for estimating the forced local oscillation. However, on comparison of mode 2 estimates by centralized and decentralized architecture, the decentralized architecture does better for both type of input signals.

\section{CONCLUSION}

A mode-meter application was tested and validated in a real-time experimental environment using PMU data streams when developed in two different architectures: centralized and

TABLE V

STATISTICAL ANALYSIS OF DECENTRALIZED MODE ESTIMATION USING VOLTAGE ANGLE DIFFERENCE FROM PMU3 AND PMU4 SIGNALS

\begin{tabular}{ccccccccc}
\hline & \multicolumn{4}{c}{ Mode 1} & \multicolumn{4}{c}{ Mode 2} \\
\cline { 2 - 9 } Signal & \multicolumn{2}{c}{ Freq. (Hz) } & \multicolumn{1}{c}{ Damp. (\%) } & \multicolumn{2}{c}{ Freq. (Hz) } & \multicolumn{2}{c}{ Damp. (\%) } \\
\cline { 2 - 10 } & $\mu$ & $\sigma$ & $\mu$ & $\sigma$ & $\mu$ & $\sigma$ & $\mu$ & $\sigma$ \\
\hline \multirow{2}{*}{$3-4$} & .4175 & .0049 & 2.47 & 1.54 & 1.702 & .048 & 1.18 & 0.55 \\
\hline
\end{tabular}


decentralized. Voltage magnitude and voltage angle differences from different PMUs were tested as input signals to both architectures. It was shown that the application was able to detect the major inter-area oscillations using both architectures. It was found out that in case of the voltage magnitude signals, the decentralized architecture substantially improves the accuracy of estimates for the localized oscillations. In the case of voltage angle differences, the decentralized architecture gives marginally more accurate results than the centralized architecture. Over all, it was shown that decentralized architecture improved the accuracy of estimates for local, low amplitude oscillations in case of all the signal types.

\section{REFERENCES}

[1] R. S. Singh, H. Hooshyar and Luigi Vanfretti, “ 'In Silico' Testing of a Decentralized PMU Data-Based Power Systems Mode Estimator," in Proc. 2016 IEEE Power and Energy Society General Meeting Conf., pp. 315-320. [Online]. Available KTH Diva Portal: http://kth.diva-portal.org/ smash/get/diva2:917903/FULLTEXT01.pdf

[2] J.F. Hauer, D.J. Trudnowski, J.G. and DeSteese, "A Perspective on WAMS Analysis Tools for Tracking of Oscillatory Dynamics," in Proc. IEEE Power Engineering Society General Meeting 2007, June 2007, pp. $1-10$.

[3] J. Ning, S.A.N. Samadi, V. Venkatasubramanian, "Two-Level Ambient Oscillation Modal Estimation From Synchrophasor Measurements", in IEEE Trans. Power Systems, vol. 30, No. 6, pp. 2913-2922, Aug. 2015.

[4] Identification of Electromechanical Modes in Power Systems, IEEE Task Force Report, Jun. 2012

[5] L. Vanfretti, V. H. Aarstrand, M. S. Almas, V. S. Peri and J. O. Gjerde, "A software development toolkit for real-time synchrophasor applications" in Proc. PowerTech (POWERTECH), 2013 IEEE Grenoble, Grenoble, 2013, pp. 1-6.

[6] Smart grid Synchrophasor SDK (S3DK) - A LabVIEW API and a RealTime Data Mediator for PMU Application Development! https://github. com/SmarTS-Lab-Parapluie/S3DK

[7] H. Hooshyar, F. Mahmood, L. Vanfretti, M. Baudette, "Specification, implementation, and hardware-in-the-loop real-time simulation of an active distribution grid", in Elsevier Journal of Sustainable Energy, Grids and Networks (SEGAN), Volume 3, September 2015, Pages 36-51.

[8] Active Distribution Network Power System Model developed in the FP7 IDE4L Project by KTH SmarTS Lab. https://github.com/SmarTS-Lab/ FP7-IDE4L-KTHSmarTSLab-ADN-RTModel 